\title{
Sub-Linear Root Detection, and New Hardness Results, for Sparse Polynomials Over Finite Fields
}

\author{
Jingguo $\mathrm{Bi}^{*}$ \\ Institute for Advanced Study \\ Tsinghua University \\ Beijing, China \\ jingguobi@mail.tsinghua.edu.cn
}

\author{
J. Maurice Rojas \\ Department of Mathematics \\ Texas A\&M University College \\ Station, Texas 77843-3368, \\ USA. \\ rojas@math.tamu.edu
}

\begin{abstract}
We present a deterministic $2^{O(t)} q^{\frac{t-2}{t-1}+o(1)}$ algorithm to decide whether a univariate polynomial $f$, with exactly $t$ monomial terms and degree $<q$, has a root in $\mathbb{F}_{q}$. Our method is the first with complexity sub-linear in $q$ when $t$ is fixed. We also prove a structural property for the nonzero roots in $\mathbb{F}_{q}$ of any $t$-nomial: the nonzero roots always admit a partition into no more than $2 \sqrt{t-1}(q-1)^{\frac{t-2}{t-1}}$ cosets of two subgroups $S_{1} \subseteq S_{2}$ of $\mathbb{F}_{q}^{*}$. This can be thought of as a finite field analogue of Descartes' Rule. A corollary of our results is the first deterministic sub-linear algorithm for detecting common degree one factors of $k$-tuples of $t$-nomials in $\mathbb{F}_{q}[x]$ when $k$ and $t$ are fixed.

When $t$ is not fixed we show that, for $p$ prime, detecting roots in $\mathbb{F}_{p}$ for $f$ is $\mathbf{N P}$-hard with respect to $\mathbf{B P P}$ reductions. Finally, we prove that if the complexity of root detection is sub-linear (in a refined sense), relative to the straight-line program encoding, then NEXP $\nsubseteq \mathbf{P} /$ poly.
\end{abstract}

\section{Categories and Subject Descriptors}

F.2.1 [Numerical Algorithms and Problems]: Computations in finite fields; F.1.3 [Complexity Measures and Classes]: Reducibility and completeness

\section{Keywords}

Solvability, sparse polynomial, finite fields, NP-hardness, gcd, square-free, discriminant, resultant

\footnotetext{
*J.B. was partially supported by: 973 Program (Grant 2013CB834205) and NSF of China under grants No.61133013 \& 61272035.

$\dagger^{\dagger}$ Q.C. was partially supported by 973 Program (Grant 2013CB834201) and NSF under grants CCF-0830522 and CCF-0830524.

†J.M.R. was partially supported by NSF MCS grant DMS0915245, DOE ASCR grant DE-SC0002505, and Sandia National Laboratories.
}

Permission to make digital or hard copies of all or part of this work for personal or classroom use is granted without fee provided that copies are not made or distributed for profit or commercial advantage and that copies bear this notice and the full citation on the first page. To copy otherwise, to republish, to post on servers or to redistribute to lists, requires prior specific permission and/or a fee.

ISSAC'13, June 26-29, 2013, Boston, Massachusetts, USA

Copyright 2013 ACM 978-1-4503-2059-7/13/06 ...\$15.00.

\section{INTRODUCTION}

The solvability of univariate sparse polynomials is a fundamental problem in computer algebra, and an important precursor to deep questions in polynomial system solving and circuit complexity. Cucker, Koiran, and Smale [CKS99] found a polynomial-time algorithm to find all integer roots of a univariate polynomial $f$ in $\mathbb{Z}[x]$ with exactly $t$ terms, i.e., a univariate t-nomial. Shortly afterward, H. W. Lenstra, Jr. [Len99] gave a polynomial-time algorithm to compute all factors of fixed degree over an algebraic extension of $\mathbb{Q}$ of fixed degree (and thereby all rational roots). Independently, Kaltofen and Koiran [KK05] and Avendano, Krick, and Sombra [AKS07] extended this to finding bounded-degree factors of sparse polynomials in $\mathbb{Q}[x, y]$ in polynomial-time. Unlike the famous LLL factoring algorithm [LLL82], the complexity of the algorithms from [CKS99, Len99, KK05, AKS07] was relative to the sparse encoding (cf. Definition 2.1 of Section 2 below) and thus polynomial in $t+\log \operatorname{deg} f$.

Changing the ground field dramatically changes the complexity. For instance, while polynomial-time algorithms are now known for detecting real roots for trinomials in $\mathbb{Z}[x]$ [RY05, BRS09], no polynomial-time algorithm is known for tetranomials [BHPR11]. Also, detecting $p$-adic rational roots for trinomials in $\mathbb{Z}[x]$ was only recently shown to lie in NP (for a fixed prime $p$ ), as was NP-hardness with respect to ZPP-reductions for $t$-nomials when neither $t$ nor $p$ are fixed [AIRR12, Thm. 1.4 \& Cor. 1.5].

Here, we focus on the complexity of detecting solutions of univariate $t$-nomials over finite fields.

\subsection{Main Results and Related Work}

While deciding the existence of a $d^{\text {th }}$ root of an element of the $q$-element field $\mathbb{F}_{q}$ is doable in time polynomial in $\log (d)+\log q$ (see, e.g., [BS96, Thms. 5.6.2 \& 5.7.2, pg. 109]), detecting roots for a trinomial equation $a+b x^{d_{0}}+c x^{d}=0$ with $d>d_{0}>0$ within time sub-linear in $d$ and $q$ is already a mystery. (Erich Kaltofen and David A. Cox independently asked about such polynomial-time algorithms around 2003 [Kal03, Cox04].) We make progress on a natural extension of this question. In what follows, we use $|S|$ for the cardinality of a set $S$.

THEOREM 1.1. Given any univariate t-nomial

$$
f(x):=c_{1}+c_{2} x^{a_{2}}+c_{3} x^{a_{3}}+\cdots+c_{t} x^{a_{t}} \in \mathbb{F}_{q}[x]
$$

with degree $<q$, we can decide, within $4^{t+o(1)} q^{\frac{t-2}{t-1}+o(1)} d e-$ terministic bit operations, whether $f$ has a root in $\mathbb{F}_{q}$. More-

over, letting $\delta:=\operatorname{gcd}\left(q-1, a_{2}, \ldots, a_{t}\right)$ and $\eta:=\sqrt{t-1}\left(\frac{q-1}{\delta}\right)^{\frac{t-2}{t-1}}$, the entire set of nonzero roots of $f$ in $\mathbb{F}_{q}$ is a union of 
at most $2 \eta$ cosets of two subgroups $S_{1} \subseteq S_{2}$ of $\mathbb{F}_{q}^{*}$, where $\left|S_{1}\right|=\delta,\left|S_{2}\right|$ can be determined in time $4^{t+o(1)}(\log q)^{O(1)}$ and $\left|S_{2}\right| \geq \frac{\delta^{\frac{t-2}{t-1}}(q-1)^{\frac{1}{t-1}}}{\sqrt{t-1}}$.

The degree assumption is natural since $x^{q}=x$ in $\mathbb{F}_{q}[x]$. Note also that deciding whether an $f$ as above has a root in $\mathbb{F}_{q}$ via brute-force search takes $q^{1+o(1)}$ bit operations, assuming $t$ is fixed.

The classic Descartes' Rule [SL54] implies that the number of distinct real roots of a real univariate $t$-nomial is at most $2 t-1$, regardless of the degree. At first glance, one would think that the polynomial $x^{q-1}-1 \in \mathbb{F}_{q}[x]$ immediately renders a finite field analogue impossible. On the other hand, note that the nonzero roots of any binomial form a coset of a subgroup of $\mathbb{F}_{q}^{*}$. Our first main result indicates that, over a finite field, the number of cosets needed to cover the set of nonzero roots of a sparse polynomial $f$ is much smaller than the degree of $f$. We thus obtain a finite field analogue of Descartes' Rule. We consider the new idea of counting by cosets as one of the main contributions of this paper. More to the point, Theorem 1.1 provides new structural and algorithmic information, complementing an earlier finite field analogue of Descartes' Rule [CFKLLS00, Lemma 7]. Theorem 1.1 can also be thought of as a refined, positive characteristic analogue of results of Tao and Meshulam [Tao05, Mes06] bounding the number of complex roots of unity at which a sparse polynomial can vanish (a.k.a. uncertainty inequalities over finite groups).

Note that if we pick $a_{2}, \ldots, a_{t}$ uniformly randomly in $\{-M, \ldots, M\}$ then, as $M \longrightarrow \infty$, the probability that $\operatorname{gcd}\left(a_{2}, \cdots, a_{t}\right)=1$ approaches $1 / \zeta(t-1)$ (see, e.g., [Chr56]). The latter quantity increases from $\frac{6}{\pi^{2}} \approx 0.6079$ to 1 as $t$ goes from 3 to $\infty$. Our theorem thus implies that, with "high" probability, the nonzero roots of a sparse polynomial over a finite field can be divided into two components: one component consisting of no more than $q^{c}$ (for some $c<1$ ) isolated roots, and the other component consisting of $q^{c}$ cosets of a (potentially large) subgroup of $\mathbb{F}_{q}^{*}$. Put another way, if the number of roots of a univariate $t$-nomial in $\mathbb{F}_{q}^{*}$ is much larger than $q^{\frac{t-2}{t-1}}$, then the roots must exhibit a strong multiplicative structure.

Since detecting roots over $\mathbb{F}_{q}$ is the same as detecting linear factors of polynomials in $\mathbb{F}_{q}[x]$, it is natural to ask about the complexity of factoring sparse polynomials over $\mathbb{F}_{q}[x]$. The asymptotically fastest randomized algorithm for factoring arbitrary $f \in \mathbb{F}_{q}[x]$ of degree $d$ uses $O\left(d^{1.5}+d^{1+o(1)} \log q\right)$ arithmetic operations in $\mathbb{F}_{q}[\mathrm{KU} 11]$, but no complexity bound polynomial in $t+\log (d)+\log q$ is known. (See [Ber70, CZ81, GS92, KS98, Uma08] for some important milestones, and [GP01, Kal03, vzGat06] for an extensive survey on factoring.) However, to detect roots in $\mathbb{F}_{q}$, we don't need the full power of factoring: we need only decide whether $\operatorname{gcd}\left(x^{q}-x, f(x)\right)$ has positive degree. Indeed, a consequence of our first main result is a speed-up for a variant of the latter decision problem.

Corollary 1.2. Given any univariatet-nomials $f_{1}, \ldots, f_{k}$ $\in \mathbb{F}_{q}[x]$, we can decide if $f_{1}, \ldots, f_{k}$ have a common degree one factor in $\mathbb{F}_{q}[x]$ via a deterministic algorithm with complexity $4^{k t-k+o(1)} q^{\frac{k t-k-1}{k t-k}+o(1)}$.
Corollary 1.2 (proved in Section 3.2) appears to give the first sub-linear algorithm for detecting roots of $k$-tuples of univariate $t$-nomials for $k$ and $t$ fixed.

REMARK 1.3. It is important to note that the $k=2$ case is not the same as deciding whether the gcd of two general polynomials has positive degree: the latter problem is the same as detecting common factors of arbitrary degree, or degree one factors over an extension field. Finding an algorithm for the latter problem with complexity sub-linear in $q$ is already an open problem for $k=2$ and $t \geq 3$ : see [EP05], and Theorem 1.5 and Remark 1.7 below. $\diamond$

One reason why it is challenging to attain complexity sublinear in $q$ is that detecting roots in $\mathbb{F}_{q}$ for $t$-nomials is NPhard when $t$ is not fixed, even restricting to one variable and prime $q$.

THEOREM 1.4. Suppose that, for any input $(f, p)$ with $p$ a prime and $f \in \mathbb{F}_{p}[x]$ a t-nomial of degree $<p$, one could decide whether $f$ has a root in $\mathbb{F}_{p}$ within $\mathbf{B P P}$, using $t+\log p$ as the underlying input size. Then $\mathbf{N P} \subseteq \mathbf{B P P}$.

The least $n$ making root detection in $\mathbb{F}_{p}^{n}$ be NP-hard for polynomials in $\mathbb{F}_{p}\left[x_{1}, \ldots, x_{n}\right]$ (for $p$ prime, and relative to the sparse encoding) appears to have been unknown. Theorem 1.4 thus comes close to settling this problem. Theorem 1.4 also complements an earlier result of Kipnis and Shamir proving NP-hardness for detecting roots of univariate sparse polynomials over fields of the form $\mathbb{F}_{2^{\ell}}$ [KiSha99]. Furthermore, Theorem 1.4 improves another recent NPhardness result where the underlying input size was instead the (smaller) straight-line program complexity [CHW11].

Let $\overline{\mathbb{F}}_{q}$ denote the algebraic closure of $\mathbb{F}_{q}$. A consequence of our last complexity lower bound is the hardness of detecting degenerate roots over $\mathbb{F}_{p}$ and $\overline{\mathbb{F}}_{q}$ :

TheOREM 1.5. Consider the following two problems, each with input $(f, p)$ where $p$ is a prime and $f \in \mathbb{F}_{p}[x]$ is a $t$ nomial of degree $<p$.

1. Decide whether $f$ is divisible by the square of a degree one polynomial in $\mathbb{F}_{p}[x]$.

2. Decide whether $f$ is divisible by the square of a degree one polynomial in $\overline{\mathbb{F}}_{p}[x]$.

Then, using $t+\log p$ as the underlying input size, each of these problems is NP-hard with respect to $\mathbf{B P P}$-reductions.

The NP-hardness of both problems had been previously unknown. Theorem 1.5 thus improves [KaShp99, Cor. 2] where NP-hardness (with respect to BPP-reductions) was proved for the harder variant of Problem (2) where one allows $f$ in the larger ring $\overline{\mathbb{F}}_{p}[x]$.

REMARK 1.6. Note that detecting a degenerate root for $f$ is the same as detecting a common degree one factor of $f$ and $\frac{\partial f}{\partial x}$, at least when $\operatorname{deg} f$ is less than the characteristic of the field. So an immediate consequence of Theorem 1.5 is that detecting common degree one factors in $\mathbb{F}_{p}[x]$ (resp. $\left.\overline{\mathbb{F}}_{p}[x]\right)$ for pairs of polynomials in $\mathbb{F}_{p}[x]$ is $\mathbf{N P}$-hard with respect to BPP-reductions. We thus also strengthen earlier work proving similar complexity lower bounds for detecting common degree one factors in $\mathbb{F}_{q}[x]$ (resp. $\overline{\mathbb{F}}_{q}[x]$ ) [vzGKS96, Thm. 4.11]. $\diamond$ 
REMARK 1.7. It should be noted that Problem (2) is equivalent to deciding the vanishing of univariate $\mathcal{A}$-discriminants (see [GKZ94, Ch. 12, pp. 403-408] and Definitions 2.6 and 2.8 of Section 2.2 below). While the trinomial case of Problem (2) can be done in $\mathbf{P}$ (see [AIRR12, Lemma 5.3]), we are unaware of any other speed-ups for fixed $t$. In particular, it follows immediately from Theorem 1.5 that deciding the vanishing of univariate resultants (see, e.g., [GKZ94, Ch. 12, Sec. 1, pp. 397-402] and Definition 2.6 of Section 2.2 below), of polynomials in $\mathbb{F}_{p}[x]$, is also NP-hard with respect to $\mathbf{B P P}$-reductions. $\diamond$

Our final result is a complexity separation depending on a weak tractability assumption for detecting roots of univariate polynomials given as straight-line programs (SLPs).

THEOREM 1.8. Suppose that, given any straight-line program of size $L$ representing a polynomial $f \in \mathbb{F}_{2^{\ell}}[x]$, we could decide if $f$ has a root in $\mathbb{F}_{2^{\ell}}$ within time $L^{O(1)} 2^{\ell-\omega(\log \ell)}$. Then $\mathbf{N E X P} \nsubseteq \mathbf{P} /$ poly .

One should recall that $\mathbf{N E X P} \subseteq \mathbf{P} /$ poly $\Longleftrightarrow \mathbf{N E X P}=$ MA [IKW01]. So the conditional assertion of our last theorem indeed implies a new separation of complexity classes. It may actually be the case that there is no algorithm for detecting roots in $\mathbb{F}_{2^{\ell}}$ better than brute-force search. Such a result would be in line with the Exponential Time Hypothesis [IP01] and the widely-held belief in the cryptographic community that the only way to break a well-designed block cipher is by exhaustive search.

\subsection{Highlights of Main Techniques}

Let $e$ be a positive integer such that $\operatorname{gcd}(e, q-1)=1$. If we replace $x$ by $x^{e}$ in

$$
f(x)=c_{1}+c_{2} x^{a_{2}}+c_{3} x^{a_{3}}+\cdots+c_{t} x^{a_{t}} \in \mathbb{F}_{q}[x],
$$

then we obtain

$$
f\left(x^{e}\right)=c_{1}+c_{2} x^{e a_{2}}+c_{3} x^{e a_{3}}+\cdots+c_{t} x^{e a_{t}} .
$$

These two polynomials have the same number of roots in $\mathbb{F}_{q}$ since the map from $\mathbb{F}_{q}$ to $\mathbb{F}_{q}$ given by $x \mapsto x^{e}$ is one-to-one. Now suppose that $\left(m_{2}, m_{3}, \cdots, m_{t}\right) \in \mathbb{Z}^{t-1}$ satisfies

$$
m_{2} \equiv e a_{2} \bmod q-1, \ldots, m_{t} \equiv e a_{t} \bmod q-1 .
$$

Then $f$ has a root in $\mathbb{F}_{q}$ iff the polynomial

$$
c_{1}+c_{2} x^{m_{2}}+c_{3} x^{m_{3}}+\cdots+c_{t} x^{m_{t}}
$$

has a root in $\mathbb{F}_{q}$. The key new advance needed to attain our speed-ups is a method employing recent fast algorithms for the Shortest Vector Problem (SVP) (see [MV10] and Section 2.1). In particular, our method finds a suitable $e$ that lowers the degree of any sparse polynomial in $\mathbb{F}_{q}[x]$ to a power of $q$ strictly less than 1 while still preserving solvability over $\mathbb{F}_{q}$.

Lemma 1.9. Given integers $a_{1}, \cdots, a_{t}, N$ satisfying $0<$ $a_{1}<\cdots<a_{t}<N$ and $\operatorname{gcd}\left(N, a_{1}, \cdots, a_{t}\right)=1$, one can find, within $4^{t}(t \log N)^{O(1)}$ bit operations, an integer $e$ with the following property for all $i \in\{1, \ldots, t\}$ : if $m_{i} \in$ $\{-\lfloor N / 2\rfloor, \ldots,\lceil N / 2\rceil\}$ is the unique integer congruent to $e a_{i}$ $\bmod N$ then $\left|m_{i}\right| \leq \sqrt{t} N^{1-t^{-1}}$.

We prove this lemma in Section 2.1. The lemma can be applied to the exponents of a general sparse polynomial to yield Theorem 1.1 in Section 3.1, after overcoming two potential difficulties: one can sometimes have $\operatorname{gcd}\left(q-1, a_{1}, \cdots, a_{t}\right)>$ 1 or $\operatorname{gcd}(e, q-1)>1$.

Recall that any Boolean expression of one of the following forms:

$(\diamond) y_{i} \vee y_{j} \vee y_{k}, \neg y_{i} \vee y_{j} \vee y_{k}, \neg y_{i} \vee \neg y_{j} \vee y_{k}, \neg y_{i} \vee \neg y_{j} \vee \neg y_{k}$, with $i, j, k \in[3 n]$, is a 3CNFSAT clause. A satisfying assigment for an arbitrary Boolean formula $B\left(y_{1}, \ldots, y_{n}\right)$ is an assigment of values from $\{0,1\}$ to the variables $y_{1}, \ldots, y_{n}$ which makes the equality $B\left(y_{1}, \ldots, y_{n}\right)=1$ true. $^{1}$

A key construction behind the proofs of Theorems 1.4 and 1.5 in Section 4 is a highly structured randomized reduction from 3CNFSAT to detecting roots of univariate polynomial systems over finite fields. In particular, the finite fields arising in this reduction have cardinality coming from a very particular family of prime numbers. (See Definition 2.1 from Section 2 for our definition of input size.)

THEOREM 1.10. Given any 3CNFSAT instance $B\left(y_{1}, \ldots, y_{n}\right)$ in $n \geq 4$ variables with $k$ clauses, there is a (Las Vegas) randomized polynomial-time algorithm that produces positive integers $c, p_{1}, \ldots, p_{n}$ and a $k$-tuple of polynomials $\left(f_{1}, \ldots, f_{k}\right)$ $\in(\mathbb{Z}[x])^{k}$ with the following properties:

1. $c \geq 11$ and $\log \left(c p_{1} \cdots p_{n}\right)=n^{O(1)}$.

2. $p_{1}, \ldots, p_{n}$ is an increasing sequence of primes and $p:=$ $1+c p_{1} \cdots p_{n}$ is prime.

3. For all $i, f_{i}$ is monic, $f_{i}(0) \neq 0$, $\operatorname{deg} f_{i}<p_{1} \cdots p_{n}$, and $\operatorname{size}\left(f_{i}\right)=n^{O(1)}$.

4. For all $i$, the $\bmod p$ reduction of $f_{i}$ has exactly $\operatorname{deg} f_{i}$ distinct roots in $\mathbb{F}_{p}$.

5. B has a satisfying assignment if and only if the mod $p$ reduction of $\left(f_{1}, \ldots, f_{k}\right)$ has a root in $\mathbb{F}_{p}$.

Theorem 1.10 is based on an earlier reduction of Plaisted involving complex roots of unity [Pla84, Sec. 3, pp. 127129] and was refined into the form below in [AIRR12, Secs. $6.2-6.3] .^{2}$

We now review some additional background necessary for our proofs.

\section{BACKGROUND}

Our main notion of input size essentially reduces to how long it takes to write down monomial term expansions, a.k.a. the sparse encoding.

DeFinition 2.1. For any polynomial $f \in \mathbb{Z}\left[x_{1}, \ldots, x_{n}\right]$ written $f(x)=\sum_{i=1}^{t} c_{i} x_{1}^{a_{1, i}} \cdots x_{n}^{a_{n, i}}$, we define

$$
\operatorname{size}(f):=\sum_{i=1}^{t} \log _{2}\left[\left(2+\left|c_{i}\right|\right)\left(2+\left|a_{1, i}\right|\right) \cdots\left(2+\left|a_{n, i}\right|\right)\right] .
$$

Also, when $F:=\left(f_{1}, \ldots, f_{k}\right)$, we define $\operatorname{size}(F):=\sum_{i=1}^{k} \operatorname{size}\left(f_{i}\right)$. $\diamond$

The definition above is also sometimes known as the sparse size of a polynomial. Note that $\operatorname{size}(c)=O(\log |c|)$ for any integer $c$.

\footnotetext{
${ }^{1}$ We respectively identify 0 and 1 with "False" and "True". ${ }^{2}$ [AIRR12] in fact contains a version of Theorem 1.10 with $c \geq 2$, but $c \geq 11$ can be attained by a trivial modification of the proof there.
} 
A useful fact, easily obtainable from the famous SchwartzZippel Lemma is that systems of univariate polynomial equations can, at the expense of some randomization, be reduced to pairs of univariate equations. (See [GH93] for a multivariate version.)

LEMma 2.2. Given any prime power $q$ and $f_{1}, \ldots, f_{k} \in$ $\mathbb{F}_{q}[x]$, let $Z\left(f_{1}, \ldots, f_{k}\right)$ denote the set of solutions of $f_{1}=$ $\cdots=f_{k}=0$ in $\overline{\mathbb{F}}_{q}$. Also let $d:=\max _{i} \operatorname{deg} f_{i}$. Then at least a fraction of $1-\frac{d}{q}$ of the $\left(u_{2}, \ldots, u_{k}\right) \in \mathbb{F}_{q}^{k-1}$ satisfy $Z\left(f_{1}, \ldots, f_{k}\right)=Z\left(f_{1}, u_{2} f_{2}+\cdots+u_{k} f_{k}\right)$.

REMARK 2.3. For this lemma to yield a high-probability reduction from $k \times 1$ systems to $2 \times 1$ systems, we will of course need to assume that $d$ is a small constant fraction of $q$. This will indeed be the case in our upcoming applications.

Let us now observe the following complexity bound for root detection for (not necessarily sparse) polynomials over finite fields.

Proposition 2.4. Given any polynomial $f \in \mathbb{F}_{q}[x]$ of degree $d$ and $N \mid(q-1)$, we can decide within $d^{1+o(1)}(\log q)^{2+o(1)}$ deterministic bit operations whether $f$ has a root in the order $N$ subgroup of $\mathbb{F}_{q}^{*}$.

Since detecting roots for $f$ as above is the same as deciding whether $\operatorname{gcd}\left(x^{N}-1, f(x)\right)$ has positive degree, the complexity bound above can be attained as follows: compute $r(x):=x^{N} \bmod f(x)$ via recursive squaring [BS96, Thm. 5.4.1, pg. 103], and then compute $\operatorname{gcd}(r(x)-1, f(x))$ in time $d^{1+o(1)}(\log q)^{1+o(1)}$ via the Knuth-Schönhage algorithm [BCS97, Ch. 3].

\subsection{Geometry of Numbers for Speed-Ups}

Recall that a lattice in $\mathbb{R}^{m}$ is the set $\mathcal{L}\left(\mathbf{b}_{\mathbf{1}}, \ldots, \mathbf{b}_{\mathbf{d}}\right)=$ $\left\{\sum_{i=1}^{d} x_{i} \mathbf{b}_{\mathbf{i}} \mid x_{i} \in \mathbb{Z}\right\}$ of all integral combinations of $d$ linearly independent vectors $\mathbf{b}_{\mathbf{1}}, \ldots, \mathbf{b}_{\mathbf{d}} \in \mathbb{R}^{m}$. The integers $d$ and $m$ are respectively called the rank and dimension of the lattice. The determinant $\operatorname{det}(\mathcal{L})$ of the lattice $\mathcal{L}$ is the volume of the $d$-dimensional parallelepiped spanned by the origin and the vectors of any $\mathbb{Z}$-basis for $\mathcal{L}$. Any lattice can be conveniently represented by a $d \times m$ matrix $\mathbf{B}$ with rows $\mathbf{b}_{\mathbf{1}}, \ldots, \mathbf{b}_{\mathbf{d}}$. The determinant of the lattice $\mathcal{L}$ can then be computed as $\operatorname{det}(\mathcal{L}(\mathbf{B}))=\sqrt{\operatorname{det}\left(\mathbf{B B}^{\top}\right)}$.

Let $\|\cdot\|$ denote the Euclidean norm on $\mathbb{R}^{n}$ for any $n$. Perhaps the most famous computational problem on lattices is the (exact) Shortest Vector Problem (SVP): Given a basis of a lattice $\mathcal{L}$, find a non-zero vector $\mathbf{u} \in \mathcal{L}$, such that $\|\mathbf{v}\| \geq$ $\|\mathbf{u}\|$ for any vector $\mathbf{v} \in \mathcal{L} \backslash \mathbf{0}$. The following is a well-known upper bound on the shortest vector length in lattice $\mathcal{L}$.

Minkowski's Theorem. Any lattice $\mathcal{L}$ of rank $d$ contains a non-zero vector $\mathbf{v}$ with $\|\mathbf{v}\| \leq \sqrt{d} \operatorname{det}(\mathcal{L})^{1 / d}$.

Given a lattice with rank $d$, the celebrated $L L L$ algorithm [LLL82] can find, in time polynomial in the bit-size of a given basis for $\mathcal{L}$, a vector whose length is at most $2^{\frac{d}{2}}$ times the length of the shortest nonzero vector in $\mathcal{L}$. An algorithm with arithmetic complexity $d^{O(1)} 4^{d}$, proposed in [MV10, Sec. 5] by Micciancio and Voulgaris, is currently the fastest deterministic algorithm for solving SVP. (See [Ngu11] for a survey of other SVP algorithms.)
Let us now prepare for our degree-lowering tricks. First, we construct the lattice $\mathcal{L}$ spanned by the rows of matrix $\mathbf{B}$, where

$$
\mathbf{B}=\left[\begin{array}{cccc}
a_{1} & a_{2} & \cdots & a_{t} \\
N & 0 & \cdots & 0 \\
0 & N & \cdots & 0 \\
\vdots & \vdots & \ddots & 0 \\
0 & 0 & \cdots & N
\end{array}\right]
$$

Letting $\mathbf{v}:=\left(m_{1}, m_{2}, \cdots, m_{t}\right)$ be the shortest vector of lattice $\mathcal{L}$, there then clearly exists an integer $e$ such that $e a_{1} \equiv$ $m_{1}, \ldots, e a_{t} \equiv m_{t} \bmod N$. (In fact, $e$ is merely the coefficient of $\left(a_{1}, \ldots, a_{t}\right)$ in the underlying linear combination defining v.) Most importantly, the factorization of $\operatorname{det}(\mathcal{L})$ is rather restricted when the $a_{i}$ are relatively prime.

$$
\text { Lemma 2.5. If } \operatorname{gcd}\left(N, a_{1}, \ldots, a_{t}\right)=1 \text { then } \operatorname{det}(\mathcal{L}) \mid N^{t-1} \text {. }
$$

Proof: Since the rows of $\mathbf{B}$ do not form a basis for the lattice $\mathcal{L}$, one can not use the formula $\operatorname{det}(\mathcal{L}(\mathbf{B}))=\sqrt{\operatorname{det}\left(\mathbf{B B}^{\top}\right)}$ to calculate the determinant of $\mathcal{L}$. Let $\mathcal{L}_{i}$ denote the sublattice of $\mathcal{L}$ generated by all rows of $\mathbf{B}$ save the $i$ th row. Clearly then, $\operatorname{det}(\mathcal{L}) \mid \operatorname{det}\left(\mathcal{L}_{i}\right)$ for all $i$. Moreover, we have $\operatorname{det}\left(\mathcal{L}_{1}\right)=$ $N^{t}$ and, via minor expansion from the $i^{\text {th }}$ column of $\mathbf{B}$, we have $\operatorname{det}\left(\mathcal{L}_{i+1}\right)=a_{i} N^{t-1}$ for all $i \in\{1, \ldots, t\}$. So $\operatorname{det}(\mathcal{L})$ divides $a_{1} N^{t-1}, \ldots, a_{t} N^{t-1}$ and we are done.

We are now ready to prove Lemma 1.9.

Proof of Lemma 1.9: From Lemma 2.5 and Minkowski's theorem, there exists a shortest vector $\mathbf{v}$ of $\mathcal{L}$ satisfying $\|\mathbf{v}\| \leq \sqrt{t} N^{1-t^{-1}}$. By invoking the exact SVP algorithm from [MV10] we can then find the shortest vector $\mathbf{v}$ in time $4^{t}(t \log N)^{O(1)}$. Let $\mathbf{v}:=\left(m_{1}, \ldots, m_{t}\right)$. Clearly, by shortness, we may assume $\left|m_{i}\right| \leq N / 2$ for all $i \in\{1, \ldots, t\}$. (Otherwise, we would be able to reduce $m_{i}$ in absolute value by subtracting a suitable row of the matrix $\mathbf{B}$ from $\mathbf{v}$.) Also, by construction, there is an $e$ such that $e a_{i} \equiv m_{i} \bmod N$ for all $i \in\{1, \ldots, t\}$.

\subsection{Resultants, $\mathcal{A}$-discriminants, and Square- Freeness}

Let us first recall the classical univariate resultant.

Definition 2.6. (See, e.g., [GKZ94, Ch. 12, Sec. 1, pp. 397-402].) Suppose $f(x)=a_{0}+\cdots+a_{d} x^{d}$ and $g(x)=b_{0}+\cdots+b_{d^{\prime}} x^{d^{\prime}}$ are polynomials with indeterminate coefficients. We define their Sylvester matrix to be the $\left(d+d^{\prime}\right) \times\left(d+d^{\prime}\right)$ matrix

$$
\left.\mathcal{S}_{\left(d, d^{\prime}\right)}(f, g):=\left[\begin{array}{cccccc}
a_{0} & \cdots & a_{d} & 0 & \cdots & 0 \\
& \ddots & & & \ddots & \\
0 & \cdots & 0 & a_{0} & \cdots & a_{d} \\
b_{0} & \cdots & b_{d^{\prime}} & 0 & \cdots & 0 \\
& \ddots & & & \ddots & \\
0 & \cdots & 0 & b_{0} & \cdots & b_{d^{\prime}}
\end{array}\right]\right\} d \text { rows }
$$

and their Sylvester resultant to be

$$
\operatorname{Res}_{\left(d, d^{\prime}\right)}(f, g):=\operatorname{det} \mathcal{S}_{\left(d, d^{\prime}\right)}(f, g) . \diamond
$$

Lemma 2.7. Following the notation of Definition 2.6, assume $f, g \in K[x]$ for some field $K$, and that $a_{d}$ and $b_{d^{\prime}}$ are not both 0 . Then $f=g=0$ has a root in the 
algebraic closure of $K$ if and only if $\operatorname{Res}_{\left(d, d^{\prime}\right)}(f, g)=0$. More precisely, we have $\operatorname{Res}_{\left(d, d^{\prime}\right)}(f, g)=a_{d}^{d^{\prime}} \prod_{f(\zeta)=0} g(\zeta)$, where the product counts multiplicity.

The lemma is classical: see, e.g., [GKZ94, Ch. 12, Sec. 1, pp. 397-402], [RS02, pg. 9], and [BPR06, Thm. 4.16, pg. 107] for a more modern treatment.

We may now define a refinement of the classical discriminant.

Definition 2.8. (See also [GKZ94, Ch. 12, pp. 403-408].) Let $\mathcal{A}:=\left\{a_{1}, \ldots, a_{t}\right\} \subset \mathbb{N} \cup\{0\}$ and $f(x):=\sum_{i=1}^{t} c_{i} x^{a_{i}}$, where $0 \leq a_{1}<\cdots<a_{t}$ and the $c_{i}$ are indeterminates. We then define the $\mathcal{A}$-discriminant of $f, \Delta_{\mathcal{A}}(f)$, to be

$$
\operatorname{Res}_{\left(\bar{a}_{t}, \bar{a}_{t}-\bar{a}_{2}\right)}\left(\bar{f}, \frac{\partial \bar{f}}{\partial x} / x^{\bar{a}_{2}-1}\right) / c_{t}^{\bar{a}_{t}-\bar{a}_{t-1}},
$$

where $\bar{a}_{i}:=\left(a_{i}-a_{1}\right) / g$ for all $i, \bar{f}(x):=\sum_{i=1}^{t} c_{i} x^{\bar{a}_{i}}$, and $g:=\operatorname{gcd}\left(a_{2}-a_{1}, \ldots, a_{t}-a_{1}\right) . \diamond$

REMARK 2.9. Note that when $\mathcal{A}=\{0, \ldots, d\}$ we have $\Delta_{\mathcal{A}}(f)=\operatorname{Res}_{(d, d-1)}\left(f, f^{\prime}\right) / c_{d}$, i.e., for dense polynomials, the $\mathcal{A}$-discriminant agrees with the classical discriminant. $\diamond$

LEMma 2.10. Suppose $p$ is any prime and $f, g \in \mathbb{F}_{p}[x]$ are relatively prime polynomials satisfying $f(0) g(0) \neq 0$, $d:=\operatorname{deg} g \geq \operatorname{deg} f$, and $p>d$. Then the polynomial $f+a g$ is square-free for at least a fraction of $1-\frac{2 d-1}{p}$ of the $a \in \mathbb{F}_{p}$.

Proof: For $2 d-1 \geq p$ the lemma is vacuous, so let us assume $2 d-1<p$. Note also that the polynomial $f+a g$ is irreducible in $\mathbb{F}_{p}[x, a]$, since $f$ and $g$ have no common factors in $\mathbb{F}_{p}[x]$. The splitting field $L \varsubsetneqq \overline{\mathbb{F}_{p}(a)}$ of $f(x)+a g(x)$ must have degree $\left[L: \mathbb{F}_{p}(a)\right]$ dividing $(\operatorname{deg} f)$ !. Since $\operatorname{deg} f \leq d<p, p$ can not divide $\left[L: \mathbb{F}_{p}(a)\right]$ and thus $L$ is a separable extension of $\mathbb{F}_{p}(a)$, i.e., $f+a g$ has no degenerate roots in $\overline{\mathbb{F}_{p}(a)}$. So the classical discriminant of $f+a g$ (where the coefficients are considered as polynomials in $a$ ) is a polynomial in $a$ that is not identically zero. Furthermore, from Definition 2.6, $\operatorname{Res}_{(d, d-1)}\left(f+a g, f^{\prime}+a g^{\prime}\right) \in \mathbb{F}_{p}[a]$ has degree at most $d+d-1=2 d-1$. So by Lemma 2.2 , the classical discriminant of $f+a g$ is non-zero for at least $1-\frac{2 d-1}{p}$ of the $a \in \mathbb{F}_{p}$. Thanks to Lemma 2.7, we thus obtain that $f+a g$ is square-free for at least a fraction of $1-\frac{2 d-1}{p}$ of the $a \in \mathbb{F}_{p}$.

REMARK 2.11. Just as for Lemma 2.2, we will need to assume that $d$ is a small constant fraction of $q$ for Lemma 2.10 to be useful. This will indeed be the case in our upcoming applications. $\diamond$

A stronger assertion, satisfied on a much smaller set of $a$, was observed earlier in the proof of Theorem 1 of [KaShp99]. For our purposes, easily finding an $a$ with $f+a g$ square-free will be crucial.

\section{FASTER ROOT DETECTION: PROVING THEOREM 1.1 AND COROLLARY 1.2}

\subsection{Proving Theorem 1.1}

Before proving Theorem 1.1, let us first prove a result that will in fact enable sub-linear root detection in arbitrary subgroups of $\mathbb{F}_{q}^{*}$.

Lemma 3.1. Given a finite field $\mathbb{F}_{q}$ and the polynomials $(\star \star \star) \quad x^{N}-1$ and $c_{1}+c_{2} x^{a_{2}}+\cdots+c_{t} x^{a_{t}}$, in $\mathbb{F}_{q}[x]$ with $0<a_{2}<\cdots<a_{t}<N, \operatorname{gcd}\left(N, a_{2}, \cdots, a_{t}\right)=1$, $c_{i} \neq 0$ for all $i$, and $N \mid(q-1)$, there exists a deterministic $q^{1 / 4}(\log q)^{O(1)}+4^{t}(t \log N)^{O(1)}+t^{\frac{1}{2}+o(1)} N^{\frac{t-2}{t-1}+o(1)}(\log q)^{2+o(1)}$ algorithm to decide whether these two polynomials share a root in $\mathbb{F}_{q}$. Furthermore, for some $\delta^{\prime} \mid N$ with $\delta^{\prime} \leq \sqrt{t-1} N^{\frac{t-2}{t-1}}$ and $\gamma \in\left\{1, \ldots, \delta^{\prime}\right\}$, the set of roots of $(\star \star \star)$ is equal to the union of a set of cardinality at most $2 \gamma \sqrt{t-1} N^{\frac{t-2}{t-1}} / \delta^{\prime}$ and the union of $\delta^{\prime}-\gamma$ cosets of a subgroup of $\mathbb{F}_{q}^{*}$ of order $N / \delta^{\prime}$.

Proof of Lemma 3.1: By Lemma 1.9 we can find an integer $e$ such that, if $m_{2}, \ldots, m_{t}$ are the unique integers in the range $[-\lfloor N / 2\rfloor,\lceil N / 2\rceil]$ respectively congruent to $e a_{2}, \ldots, e a_{t}$, then $\left|m_{i}\right|<\sqrt{t-1} N^{\frac{t-2}{t-1}}$ for each $i \in\{2, \ldots, t\}$. Thanks to [MV10], this takes $4^{t}(t \log N)^{O(1)}$ deterministic bit operations. By [Shp96], we can then find a generator $\sigma$ of $\mathbb{F}_{q}^{*}$ within $q^{1 / 4}(\log q)^{O(1)}$ bit operations. For any $\tau \in \mathbb{F}_{q}^{*}$, let $\langle\tau\rangle$ denote the multiplicative subgroup of $\mathbb{F}_{q}^{*}$ generated by $\tau$.

Now, $x^{N}-1$ vanishing is the same as $x \in\left\langle\sigma^{\frac{q-1}{N}}\right\rangle$ since $N \mid(q-1)$. Let $\zeta_{N}:=\sigma^{\frac{q-1}{N}}$ and define $\delta^{\prime}:=\operatorname{gcd}(e, N)$. If $\delta^{\prime}=1$ then the map from $\left\langle\zeta_{N}\right\rangle$ to $\left\langle\zeta_{N}\right\rangle$ given by $x \mapsto x^{e}$ is one-to-one. So finding a solution for $(\star \star \star)$ is equivalent to finding $x \in\left\langle\zeta_{N}\right\rangle$ such that $c_{1}+c_{2} x^{e a_{2}}+\cdots+c_{t} x^{e a_{t}}=0$. Thanks to Lemma 1.9, the last equation can be rewritten as the lower degree equation $c_{1}+c_{2} x^{m_{2}}+\cdots+c_{t} x^{m_{t}}=0$, and we may conclude our proof by applying Proposition 2.4.

However, we may have $\delta^{\prime}>1$. In which case, the map from $\left\langle\zeta_{N}\right\rangle$ to $\left\langle\zeta_{N}\right\rangle$ given by $x \mapsto x^{e}$ is no longer one-to-one. Instead, it sends $\left\langle\zeta_{N}\right\rangle$ to a smaller subgroup $\left\langle\zeta_{N}^{\delta^{\prime}}\right\rangle$ of order $N / \delta^{\prime}$. We first bound $\delta^{\prime}$ : re-ordering monomials if necessary, we may assume that $m_{2} \neq 0$. We then obtain

$\delta^{\prime}=\operatorname{gcd}(e, N) \leq \operatorname{gcd}\left(e a_{2}, N\right)=\operatorname{gcd}\left(m_{2}, N\right) \leq\left|m_{2}\right| \leq \sqrt{t-1} N^{\frac{t-2}{t-1}}$. Any element $x \in\left\langle\zeta_{N}\right\rangle$ can be written as $\zeta_{N}^{i} z$ for some $i \in$ $\left\{0, \ldots, \delta^{\prime}-1\right\}$ and $z \in\left\langle\zeta_{N}^{\delta^{\prime}}\right\rangle$. It is then clear that $x^{N}-1=c_{1}+c_{2} x^{a_{2}}+\cdots+c_{t} x^{a_{t}}=0$ has a root in $\mathbb{F}_{q}^{*}$ if and only if there is an $i \in\left\{0, \ldots, \delta^{\prime}-1\right\}$ and a $z \in\left\langle\zeta_{N}^{\delta^{\prime}}\right\rangle$ with $c_{1}+c_{2}\left(\zeta_{N}^{i} z\right)^{a_{2}}+\cdots+c_{t}\left(\zeta_{N}^{i} z\right)^{a_{t}}=0$. Now, $\operatorname{gcd}\left(e / \delta^{\prime}, N / \delta^{\prime}\right)=$ 1. So the map from $\left\langle\zeta_{N}^{\delta^{\prime}}\right\rangle$ to $\left\langle\zeta_{N}^{\delta^{\prime}}\right\rangle$ given by $x \mapsto x^{e / \delta^{\prime}}$ is oneto-one. By the definition of the $m_{i},(\star \star \star)$ having a solution is thus equivalent to there being an $i \in\left\{0, \ldots, \delta^{\prime}-1\right\}$ and a $z \in\left\langle\zeta_{N}^{\delta^{\prime}}\right\rangle$ with $c_{1}+c_{2} \zeta_{N}^{a_{2} i} z^{m_{t} / \delta^{\prime}}+\cdots+c_{t} \zeta_{N}^{a_{t} i} z^{m_{t} / \delta^{\prime}}=0$. So define the Laurent polynomial

$$
f_{i}(z):=c_{1}+c_{2}\left(\zeta_{N}^{i}\right)^{a_{2}} z^{m_{2} / \delta^{\prime}}+\cdots+c_{t}\left(\zeta_{N}^{i}\right)^{a_{t}} z^{m_{t} / \delta^{\prime}}
$$

If $f_{i}$ is identically zero then we have found a whole set of solutions for $(\star \star \star)$ : the $\operatorname{coset} \zeta_{N}^{i}\left\langle\zeta_{N}^{\delta^{\prime}}\right\rangle$. If $f_{i}$ is not identically zero then let $\ell:=\min _{i} \min \left\{m_{i} / \delta^{\prime}, 0\right\}$. The polynomial $z^{-\ell} f_{i}(z)$ then has degree bounded from above by $2 \sqrt{t-1} N^{\frac{t-2}{t-1}} / \delta^{\prime}$. Deciding whether the pair of equations $z^{N / \delta^{\prime}}-1=z^{-\ell} f_{i}(z)=0$ has a solution for some $i$ takes deterministic time

$$
\delta^{\prime}\left(\sqrt{t-1} N^{\frac{t-2}{t-1}} / \delta^{\prime}\right)^{1+o(1)}(\log q)^{2+o(1)}
$$

applying Proposition $2.4 \delta^{\prime}$ times.

The final statement characterizing the set of solutions to $(\star \star \star)$ then follows immediately upon defining $\gamma$ to be the number of $i \in\left\{0, \ldots, \delta^{\prime}-1\right\}$ such that $f_{i}$ is not identically zero. In particular, $\gamma \geq 1$ since $\operatorname{deg} f<N$ and thus $f$ is not identically zero on the order $N$ subgroup of $\mathbb{F}_{q}^{*}$.

EXAMPLE 3.2. Consider any polynomial of the form

$$
f(x)=c_{1}+c_{2} x+c_{3} x^{2^{200}+26}+c_{4} x^{2^{200}+27} \in \mathbb{F}_{q}[x]
$$


where $q:=6\left(2^{200}+26\right)+1$ (which is a 61-digit prime) and $c_{1} c_{4} \neq 0$. Considering the lattice generated by the vectors $\left(1,2^{200}+26,2^{200}+27\right),(q-1,0,0),(0, q-1,0),(0,0, q-1)$, it is not hard to see that $(6,0,6)$ is a minimal length vector in this lattice. Moreover, $6 \cdot 1 \equiv 6,6\left(2^{200}+26\right) \equiv 0$, $6\left(2^{200}+27\right) \equiv 6 \bmod q-1$. Letting $\sigma$ be any generator of $\mathbb{F}_{q}^{*}$ it is clear that any $x \in \mathbb{F}_{q}^{*}$ can be written as $x=\sigma^{i} z$ for some $i \in\{0, \ldots, 5\}$ and $z \in \mathbb{F}_{q}^{*}$ satisfying $z^{\frac{q-1}{6}}=1$. So then, we see that solving $f(x)=0$ is equivalent to finding an $i \in\{0, \ldots, 5\}$ and $a z \in \mathbb{F}_{q}^{*}$ with

$\left(c_{1}+c_{3} \sigma^{\left(2^{200}+26\right) i}\right)+\left(c_{2} \sigma^{i}+c_{4} \sigma^{\left(2^{200}+27\right) i}\right) z^{6}=z^{\frac{q-1}{6}}-1=0$.

REMARK 3.3. Via fast randomized factoring, we can also pick out a representative from each coset of roots within essentially the same time bound. Note also that it is possible for some of the Laurent polynomials $f_{i}$ to vanish identically: the polynomial $1+x-x^{2}-x^{3}$ and the prime $q=13$, obtained by mimicking Example 3.2, provide one such example (with $\delta^{\prime}=6$ and $\left.\gamma=1\right) . \diamond$

We are now ready to prove our first main theorem.

Proof of Theorem 1.1: Let $\delta:=\operatorname{gcd}\left(q-1, a_{2}, \ldots, a_{t}\right)$ and $y=x^{\delta}$. Then the solvability of $f$ is equivalent to the solvability of the following system of equations:

$$
\begin{gathered}
c_{1}+c_{2} y^{a_{2} / \delta}+\cdots+c_{t} y^{a_{t} / \delta}=0 \\
y^{\frac{q-1}{\delta}}=1
\end{gathered}
$$

Since $\operatorname{gcd}\left(\frac{a_{1}}{\delta}, \ldots, \frac{a_{t}}{\delta}, \frac{q-1}{\delta}\right)=1$, we can solve this problem via Lemma 3.1 (with $N=\frac{q-1}{\delta}$ ), within the stated time bound. (Note that $q^{1 / 4} \leq q^{\frac{t-2}{t-1}}$ for all $t \geq 3$. Also, the computation of $\operatorname{gcd}\left(q-1, a_{2}, \ldots, a_{t}\right)$ is dominated by the other steps of the algorithm underlying Lemma 3.1.) Also, since $y^{\frac{q-1}{\delta}}=1$, each solution $y$ of the preceding $2 \times 1$ system induces exactly $\delta$ roots of $f$ in $\mathbb{F}_{q}$. So we can indeed efficiently detect roots of $f$, and the second assertion of Lemma 3.1 gives us the stated characterization of the roots of $f$. In particular, $S_{1}$ is the unique order $\delta$ subgroup of $\mathbb{F}_{q}^{*}$, and $S_{2}$ is the unique order $\frac{q-1}{\delta^{\prime}}$ subgroup of $\mathbb{F}_{q}^{*}$ (following the notation of the proof of Lemma 3.1).

\subsection{The Proof of Corollary $\mathbf{1 . 2}$}

Deciding whether 0 is a root of all the $f_{i}$ is trivial, so let us divide all the $f_{i}$ by a suitable power of $x$ so that all the $f_{i}$ have a nonzero constant term. Next, concatenate all the nonzero exponents of the $f_{i}$ into a single vector of length $T \leq k(t-1)$. Applying Lemma 1.9, and repeating our power substitution trick from our proof of Theorem 1.1, we can then reduce to the case where each $f_{i}$ has degree at most $2 \sqrt{T} q^{1-T^{-1}}$, at the expense of $4^{T}(T \log q)^{O(1)}$ deterministic bit operations.

At this stage, we then simply compute

$$
g(x):=\left(\left(\cdots\left(\operatorname{gcd}\left(\operatorname{gcd}\left(f_{1}, f_{2}\right), f_{3}\right), \ldots\right), f_{k}\right)\right.
$$

via $k-1$ applications of the Knuth-Schönhage algorithm [BCS97, Ch. 3]. This takes

$$
(k-1)\left(2 \sqrt{T} q^{1-T^{-1}}\right)^{1+o(1)}(\log q)^{1+o(1)}
$$

deterministic bit operations. We then conclude via Proposition 2.4, at a cost of $\left(2 \sqrt{T} q^{1-T^{-1}}\right)^{1+o(1)}(\log q)^{2+o(1)}$ bit operations.
Summing the complexities of our steps, we arrive at our stated complexity bound.

\section{HARDNESS IN ONE VARIABLE: PROV- ING THEOREMS 1.4, 1.5, AND 1.8}

\subsection{The Proof of Theorem 1.4}

Thanks to Theorem 1.10 we obtain an immediate ZPPreduction from 3 CNFSAT to the detection of roots in $\mathbb{F}_{p}$ for systems of univariate polynomials in $\mathbb{F}_{p}[x]$. By Lemma 2.2 and Remark 2.3 we then obtain a BPP-reduction to $2 \times 1$ systems. Let us now describe a ZPP-reduction from $2 \times 1$ systems to $1 \times 1$ systems.

Suppose $\chi \in \mathbb{F}_{q}$ is a quadratic non-residue. Clearly, the only root in $\mathbb{F}_{q}^{2}$ of the quadratic form $x^{2}-\chi y^{2}$ is $(0,0)$. So we can decide the solvability of $f_{1}(x)=f_{2}(x)=0$ over $\mathbb{F}_{q}$ by deciding the solvability of $f_{1}^{2}-\chi f_{2}^{2}$ over $\mathbb{F}_{q}$. Finding a usable $\chi$ is easily done in $\mathbf{Z P P}$ via random-sampling and polynomial-time Jacobi symbol calculation (see, e.g., [BS96, Cor. 5.7.5 \& Thm. 5.9.3, pg. 110 \& 113]).

So there is indeed a BPP-reduction from 3CNFSAT to our main problem, and we are done.

\subsection{The Proof of Theorem 1.5}

First note that the hardness of detecting common degree one factors in $\mathbb{F}_{p}[x]$ (or $\overline{\mathbb{F}}_{p}[x]$ ) for pairs of polynomials in $\mathbb{F}_{p}[x]$ follows immediately from Theorem 1.10 and Lemma 2.2: the proof of Theorem 1.4 above already tells us that there is a BPP-reduction from 3CNFSAT to detecting common roots in $\overline{\mathbb{F}}_{p}$ of pairs of polynomials in $\mathbb{F}_{p}[x]$. Thanks to Assertion (4) of Theorem 1.10, we also obtain a BPPreduction to detecting common roots, in $\mathbb{F}_{p}$ instead, for pairs of polynomials in $\mathbb{F}_{p}[x]$.

So why does this imply hardness for deciding divisibility by the square of a degree one polynomial in $\overline{\mathbb{F}}_{p}[x]\left(\right.$ or $\left.\mathbb{F}_{p}[x]\right)$ ? Assume temporarily that Problem (2) is doable in BPP. Consider then, for any $f, g \in \mathbb{F}_{p}[x]$, the polynomial $H:=$ $(f+a g)(f+b g)$ where $\{a, b\} \subset \mathbb{F}_{p}$ is a uniformly random subset of cardinality 2. Note that should $f$ and $g$ have a common factor in $\overline{\mathbb{F}}_{p}[x]$, then $H$ has a repeated factor in $\overline{\mathbb{F}}_{p}[x]$.

On the other hand, if $f$ and $g$ have no common factor, then $f+a g$ and $f+b g$ clearly have no common factors. Moreover, thanks to Lemma 2.10 and Remark 2.11, the probability that $f+a g$ and $f+b g$ are both square-free - and thus $H$ is square-free - is at least $\left(1-\frac{2 d-1}{q}\right)\left(1-\frac{2 d-2}{q}\right)$, assuming $f$ and $g$ satisfy the hypothesis of the lemma.

In other words, to test $f$ and $g$ for common factors, it's enough to check square-freeness of $H$ for random $(a, b)$.

To conclude, thanks to Theorem 1.10, the pairs of polynomials arising from our BPP-reduction from 3CNFSAT satisfy the hypothesis of Lemma 2.10. Furthermore, thanks to Assertion (1) of Theorem 1.10, our success probability is at least $\left(1-\frac{2}{11}\right)^{2} \geq \frac{2}{3}$, so we are done.

\subsection{Proving Theorem 1.8}

We will need the following proposition, due to Ryan Williams.

Proposition 4.1. [Wil10] Assume, for any Boolean circuit with $n$ inputs and size polynomial in $n$, that the Circuit Satisfiability Problem can be solved in time $2^{n-\omega(\log n)}$. Then NEXP $\nsubseteq \mathbf{P} /$ poly. 
We will also need the following lemma, which is implicit in [KiSha99]. For completeness, we supply a proof below.

LEMma 4.2. Given a Boolean circuit with d inputs and $L$ gates, we can find a straight-line program of size $L^{O(1)}$ for a polynomial $f \in \mathbb{F}_{2^{d}}[x]$ such that the circuit is satisfied if and only if $f$ has a root in $\mathbb{F}_{2^{d}}$.

Proof: A Boolean circuit can be viewed as a straight-line program using Boolean variables and Boolean operations. One can replace the Boolean operations by polynomials over $\mathbb{F}_{2}$ :

$$
\begin{gathered}
x_{1} \wedge x_{2}=x_{1} x_{2} \\
x_{1} \vee x_{2}=x_{1}+x_{2}+x_{1} x_{2} \\
\neg x_{1}=1-x_{1}
\end{gathered}
$$

Hence a straight-line program for a Boolean function of size $L$ with $d$ inputs can be converted into a straight-line program for a polynomial $f\left(x_{0}, x_{1}, \cdots, x_{d-1}\right) \in \mathbb{F}_{2}\left[x_{0}, x_{1}, \cdots, x_{d-1}\right]$ of size $O(L)$.

Let $b(x)$ be an irreducible polynomial of degree $d$ over $\mathbb{F}_{2}$. Let $\alpha$ be one root of $b(x)$. Then $\left\{1, \alpha, \alpha^{2}, \ldots, \alpha^{d-1}\right\}$ is a basis for $\mathbb{F}_{2^{d}}$ over $\mathbb{F}_{2}$. Then any element $x \in \mathbb{F}_{2^{d}}$ can be written uniquely as $x=x_{0}+x_{1} \alpha+\cdots+x_{d-1} \alpha^{d-1}$, where $x_{i} \in \mathbb{F}_{2}$ for all $i$. So we obtain the system of linear equations

$$
\left[\begin{array}{cccc}
1 & \alpha & \cdots & \alpha^{d-1} \\
1 & \alpha^{2} & \cdots & \alpha^{2(d-1)} \\
1 & \alpha^{4} & \cdots & \alpha^{4(d-1)} \\
\vdots & & & \\
1 & \alpha^{2^{d-1}} & \cdots & \alpha^{2^{d-1}(d-1)}
\end{array}\right]\left[\begin{array}{c}
x_{0} \\
x_{1} \\
x_{2} \\
\vdots \\
x_{d-1}
\end{array}\right]=\left[\begin{array}{c}
x \\
x^{2} \\
x^{4} \\
\vdots \\
x^{2^{d-1}}
\end{array}\right]
$$

The underlying matrix is Vandermonde and thus non-singular. So we can represent each $x_{i}$ as a linear combination of $x, x^{2^{1}}$, $x^{2^{2}}, \ldots, x^{2^{d-1}}$ over $\mathbb{F}_{2^{d}}$. Replacing each $x_{i}$ by the appropriate linear combination of high powers of $x$, in the SLP for $f$, we obtain our lemma.

Proof of Theorem 1.8: From Lemma 4.2, an algorithm as hypothesized in Theorem 1.8 would imply a $2^{\ell-\omega(\log \ell)}$ algorithm for any instance of the Circuit Satisfiability Problem of $\ell$ inputs and size polynomial in $\ell$. By Proposition 4.1, we would then obtain NEXP $\nsubseteq \mathbf{P} /$ poly.

\section{Acknowledgements}

We would like to thank Igor Shparlinski for insightful comments on an earlier draft of this paper. We also thank the referees for their comments.

\section{REFERENCES}

[AIRR12] Avendaño, Martíin; Ibrahim, Ashraf, Rojas, J. Maurice; and Rusek, Korben, "Faster p-adic Feasibility for Certain Multivariate Sparse Polynomials," Journal of Symbolic Computation, special issue in honor of 60th birthday of Joachim von zur Gathen, vol. 47, no. 4, pp. 454-479 (April 2012).

[AKS07] Avendaño, Martín; Krick, Teresa; and Sombra, Martín, "Factoring bivariate sparse (lacunary) polynomials," J. Complexity, vol. 23 (2007), pp. 193-216.

[BS96] Bach, Eric and Shallit, Jeff, Algorithmic Number Theory, Vol. I: Efficient Algorithms, MIT Press, Cambridge, MA, 1996.

[BHPR11] Bastani, Osbert; C. Hillar, D. Popov, and J. M. Rojas, "Randomization, Sums of Squares, and Faster
Real Root Counting for Tetranomials and Beyond," Randomization, Relaxation, and Complexity in Polynomial Equation Solving, Contemporary Mathematics, vol. 556, pp. 145-166, AMS Press, 2011.

[BPR06] Basu, Saugata; Pollack, Ricky; and Roy, Marie-Francoise, Algorithms in Real Algebraic Geometry, Algorithms and Computation in Mathematics, vol. 10, Springer-Verlag, 2006.

[Ber70] Berlekamp, Elwyn R., "Factoring polynomials over large finite fields," Math. Comp. 24, pp. 713-735 (1970).

[BRS09] Bihan, Frederic; Rojas, J. Maurice; Stella, Case E., "Faster Real Feasibility via Circuit Discriminants," proceedings of International Symposium on Symbolic and Algebraic Computation (ISSAC 2009, July 28-31, Seoul, Korea), pp. 39-46, ACM Press, 2009.

[BCS97] Bürgisser, Peter; Clausen, Michael; and Shokrollahi, M. Amin, Algebraic complexity theory, with the collaboration of Thomas Lickteig, Grundlehren der Mathematischen Wissenschaften [Fundamental Principles of Mathematical Sciences], 315, Springer-Verlag, Berlin, 1997.

[CFKLLS00] Canetti, Ran; Friedlander, John B.; Konyagin, Sergei; Larsen, Michael; Lieman, Daniel; and Shparlinski, Igor E., "On the statistical properties of Diffie-Hellman distributions," Israel J. Math. 120 (2000), pp. 23-46.

[CZ81] Cantor, David G. and Zassenhaus, Hans, "A new algorithm for factoring polynomials over finite fields," Math. Comp. 36 (1981), no. 154, pp. 587-592.

[CHW11] Cheng, Qi; Hill, Joshua E.; and Wan, Daqing, "Counting Value Sets: Algorithm and Complexity," Math ArXiV preprint 1111.1224.

[Chr56] Christopher, John, "The Asymptotic Density of Some k-Dimensional Sets," the American Mathematical Monthly, vol. 63, no. 6 (Jun.-Jul., 1956), pp. 399-401.

[Cox04] Cox, David A., personal communication, August, 2004.

[CKS99] Cucker, Felipe; Koiran, Pascal; and Smale, Steve, "A polynomial time algorithm for Diophantine equations in one variable," J. Symbolic Comput. 27 (1999), pp. 21-29.

[EP05] Emiris, Ioannis Z. and Pan, Victor, "Improved algorithms for computing determinants and resultants," J. Complexity (FOCM 2002 special issue), Vol. 21, no. 1, February 2005, pp. 43-71.

[vzGat06] von zur Gathen, Joachim, "Who was who in polynomial factorization, "Proceedings of ISSAC 2006 (B. M. Trager, ed.), pp. 2-3, ACM Press, 2006.

[vzGKS96] von zur Gathen, Joachim; Karpinski, Marek; and Shparlinski, Igor E., "Counting curves and their projections," Computational Complexity 6, no. 1 (1996/1997), pp. 64-99.

[GP01] von zur Gathen, Joachim and Panario, Daniel, "Factoring polynomials over finite fields: A survey," J. Symb. Comput., 31(1/2):3-17, 2001.

[GS92] von zur Gathen, Joachim and Shoup, Victor, "Computing Frobenius maps and factoring polynomials," Computational Complexity 2:187-224, 1992. 
[GKZ94] Gel'fand, Israel Moseyevitch; Kapranov, Misha M.; and Zelevinsky, Andrei V.; Discriminants, Resultants and Multidimensional Determinants, Birkhäuser, Boston, 1994.

[GH93] Giusti, Marc and Heintz, Joos, "La détermination des points isolés et la dimension d'une variété algébrique peut se faire en temps polynomial," Computational Algebraic Geometry and Commutative Algebra (Cortona, 1991), Sympos. Math. XXXIV, pp. 216-256, Cambridge University

[IKW01] Impagliazzo, Russell; Kabanets, Valentine; and Wigderson, Avi, "In Search of an Easy Witness: Exponential Time vs. Probabilistic Polynomial Time," Journal of Computer and System Sciences, 65(4), pp. 672-694, 2002.

[IP01] Impagliazzo, Russell and Paturi, Ramamohan, "The Complexity of k-SAT, " Journal of Computer and System Sciences, Volume 62, Issue 2, March 2001, pp. 367-375.

[JS07] Jeronimo, Gabriela and Sabia, Juan, "Computing multihomogeneous resultants using straight-line programs," J. Symbolic Comput. 42 (2007), no. 1-2, pp. 218-235.

[Kal03] Kaltofen, Erich, "Polynomial factorization: a success story," In ISSAC 2003 Proc. 2003 Internat. Symp. Symbolic Algebraic Comput. (New York, N.Y., 2003), J. R. Sendra, Ed., ACM Press, pp. 3-4.

[KK05] Kaltofen, Erich and Koiran, Pascal, "On the complexity of factoring bivariate supersparse (lacunary) polynomials, "ISSAC05, Proceedings of 2005 International Symposium Symbolic Algebraic Computation, ACM Press, New York, 2005.

[KS98] Kaltofen, Erich and Shoup, Victor, "Subquadratic-time factoring of polynomials over finite fields," Math. Comp. 67 (1998), no. 223, pp. 1179-1197.

[KaShp99] Karpinski, Marek and Shparlinski, Igor E., "On the computational hardness of testing square-freeness of sparse polynomials," Applied algebra, algebraic algorithms and error-correcting codes (Honolulu, HI, 1999), pp. 492-497, Lecture Notes in Comput. Sci., 1719, Springer, Berlin, 1999.

[KU11] Kedlaya, Kiran and Umans, C., "Fast polynomial factorization and modular composition," SIAM Journal on Computing, Vol. 40, No. 6, pp. 1767-1802, 2011.

[KiSha99] Kipnis, Aviad and Shamir, Adi, "Cryptanalysis of the HFE public key cryptosystem by relinearization," Advances in cryptology - CRYPTO '99 (Santa Barbara, CA), pp. 19-30, Lecture Notes in Comput. Sci. 1666, Springer, Berlin, 1999.

[LLL82] Lenstra, Arjen K.; Lenstra, Hendrik W., Jr.; Lovász, L., "Factoring polynomials with rational coefficients," Math. Ann. 261 (1982), no. 4, pp. 515-534.

[Len99] Lenstra (Jr.), Hendrik W., "Finding Small Degree Factors of Lacunary Polynomials," Number Theory in Progress, Vol. 1 (Zakopane-Kóscielisko, 1997), pp. 267-276, de Gruyter, Berlin, 1999.

[Mes06] Meshulam, Roy, "An uncertainty inequality for finite abelian groups, "European J. of Combinatorics, 27 (2006), pp. 63-67.

[MV10] Micciancio, D. and Voulgaris, P., "A deterministic single exponential time algorithm for most lattice problems based on voronoi cell computations," SIAM J. Computing, special issue, to appear.

[Ngu11] Nguyen, Phong Q., "Lattice Reduction Algorithms: Theory and Practice," K.G. Paterson (ed.): Eurocrypt 2011, LNCS 6632, pp. 2-6, 2011.

[Pla84] Plaisted, David A., "New NP-Hard and NP-Complete Polynomial and Integer Divisibility Problems," Theoret. Comput. Sci. 31 (1984), no. 1-2, 125-138.

[RS02] Rahman, Qazi Ibadur; and Schmeisser, Gerhard, Analytic Theory of Polynomials, Clarendon Press, London Mathematical Society Monographs 26, 2002.

[RY05] Rojas, J. Maurice and Ye, Yinyu, "On Solving Sparse Polynomials in Logarithmic Time," Journal of Complexity, special issue for the 2002 Foundations of Computation Mathematics (FOCM) meeting, February 2005, pp. 87-110.

[Sch80] Schwartz, Jacob T., "Fast Probabilistic Algorithms for Verification of Polynomial Identities," J. of the ACM 27, 701-717, 1980.

[Shp96] Shparlinski, Igor E., "On finding primitive roots in finite fields," Theoretical Computer Science, Vol. 157, Issue 2, 5 May 1996, pp. 273-275.

[SL54] Smith, David Eugene and Latham, Marcia L., The Geometry of René Descartes, translated from the French and Latin (with a facsimile of Descartes' 1637 French edition), Dover Publications Inc., New York (1954).

[Tao05] Tao, Terence, "An Uncertainty Principle for Cyclic Groups of Prime Order," Math. Res. Lett. 12 (1) (2005), pp. 121-127.

[Uma08] Umans, Christopher, "Fast polynomial factorization and modular composition in small characteristic," STOC'08, pp. 481-490, ACM, New York, 2008.

[Wil10] Williams, Ryan, "Improving exhaustive search implies superpolynomial lower bounds," in STOC, 231-240, 2010.

[Zip89] Zippel, Richard, "An explicit separation of relativised random polynomial time and relativised deterministic polynomial time, " Technical report \#965, Department of Computer Science, Cornell University, 1989. 